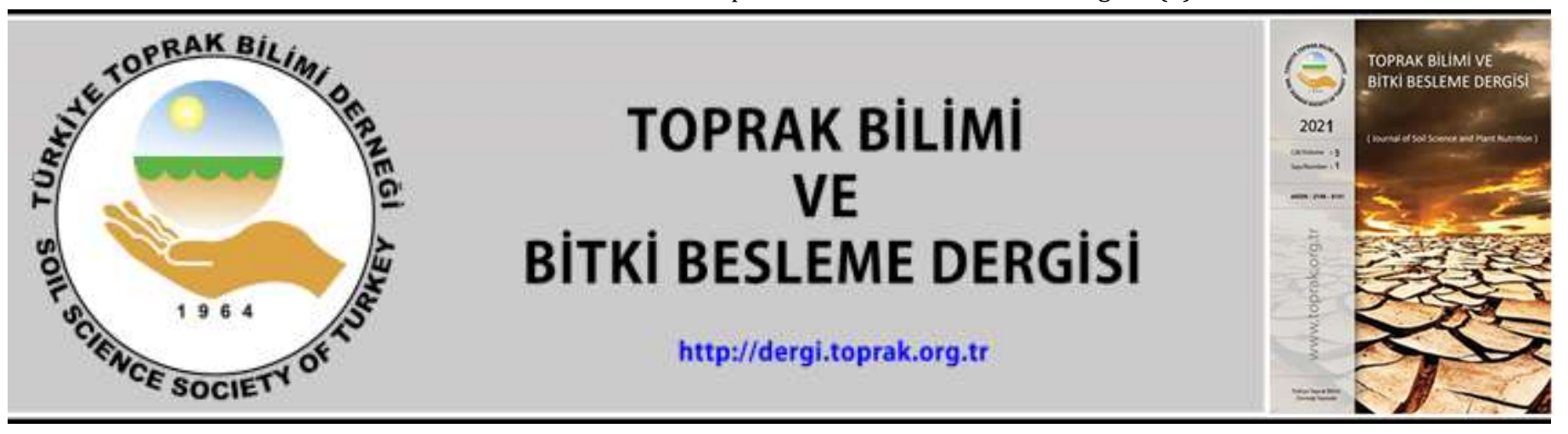

\title{
Taban suyu tablası yüksekliğinin değişimine bağlı olarak taban suyu seviyesinin matematiksel modellenmesi (Dimanverdi Ekberli*, Coşkun Gülser
}

${ }^{1}$ Ondokuz Mayıs Üniversitesi Ziraat Fakültesi Toprak Bilimi ve Bitki Besleme Bölümü, Samsun

\section{Özet}

Tarım alanlarında sulama suyu miktarının düzenlenmesinde, bitki-kök bölgesi tuzlașmasının önlenmesinde, drenaj sisteminin planlanmasında taban suyu derinliği ve seviyesinin, taban suyu tablasının değişiminin değerlendirilmesi gerekir. Bu çalışmada, Dupuit yaklaşımı ve Darcy yasasına bağlı olarak elde edilen Boussinesq denkleminin (doğrusal olmayan difüzyon denklemin), harmonik sınır koşuluna bağlı çözümüne göre taban suyu tablası ve seviyesinin değişimleri incelenmiştir. Taban suyu tablası ve seviyesinin mesafe ve zamana bağlı olduğu teorik olarak gösterilmiştir. Taban suyu tablası ve seviyesinin maksimum değişimleri sırasıyla $0.123 \mathrm{~m}$ ve $2.123 \mathrm{~m}$ olarak, $0.5 \mathrm{~m}$ mesafede ve 2 . saatte hesaplanmıștır. Minimum değișimler ise sirasıyla $-0.006 \mathrm{~m}$ ve 1.994 m olarak 2.5 m mesafede ve 2 . saatte belirlenmiștir. Taban suyu tablasının dalgalanma amplitütünün eksponansiyel olarak değiştiği ve $x>2$ m mesafede dalgalanmanın "sönme" sürecine yaklaştı̆̆ belirlenmiştir.

Anahtar Kelimeler: Taban suyu tablası, taban suyu seviyesi, Boussinesq denklemi, amplitüt, mesafe, zaman

\section{Mathematical modeling of the groundwater level depending on the change of groundwater table height}

\begin{abstract}
It is necessary to evaluate the change of the ground water table, the depth and level of groundwater for regulating the amount of irrigation water in the agricultural areas, preventing salinization in the plant-root region, and planning the drainage system. In this study, the variation of ground water table and its level depending on solution according to harmonic boundary condition of the Boussinesq equation (nonlinear diffusion equation), derived from the Dupuit approximation and the Darcy law, were investigated. It has been theoretically shown that the ground water table and its level depend on distance and time. The maximum variations of ground water table and its level were calculated as $0.123 \mathrm{~m}$ and $2.123 \mathrm{~m}$, at a distance of $0.5 \mathrm{~m}$ and at the $2^{\text {nd }}$ hour, respectively. The minimum changes were determined as $-0.006 \mathrm{~m}$ and $1.994 \mathrm{~m}$ at a distance of $2.5 \mathrm{~m}$ and at the $2^{\text {nd }}$ hour, respectively. It has been determined that the fluctuation amplitude of the groundwater table is exponentially changed and the fluctuation in $\mathrm{x}>2 \mathrm{~m}$ distance approaches the "damping" process.
\end{abstract}

Keywords: Groundwater table, groundwater level, Boussinesq equation, amplitude, distance, time

(C) 2021 Türkiye Toprak Bilimi Derneği. Her Hakkı Saklıdır

\section{Giriş}

Farklı iklim ve toprak koşullarına sahip bölgelerde tarım topraklarının su-tuz, besin vb. rejimlerinin düzenlenmesi; yıkama ve sulama suyu normlarının hesaplanması, tuzlanmanın önlenmesi gibi problemlerin çözümü, taban suyu derinliğinin ve taban suyu tablasının değişimine bağlı olarak taban suyu seviyesinin belirlenmesini gerektirmektedir. Taban suyu tablasının doyurduğu toprak seviyesinde aşağıdan yukarı doğru olan su hareketi, topraktaki doymamış su hareketine etki yapan faktörlerdendir. Sulama ve ylkama uygulamaları, aşırı yağış miktarı, bölgenin hidrojeolojik koşulları gibi faktörler taban suyu tablasının değişimine, dolayısıyla taban suyu seviyesine önemli düzeyde etki yapmaktadır. Arazi koşullarında drenaj çeşitlerinin belirlenmesinde, diğer faktörlerle beraber, taban suyu derinliği ve seviyesinin, taban suyu tablasının değişiminin değerlendirilmesi gerekir.

\section{* Sorumlu yazar:}

Tel. : $\quad 03623121919$

E-posta : iman@omu.edu.tr
Geliş Tarihi Kabul Tarihi
e-ISSN

2146-8141

DOI : $10.33409 /$ tbbbd.798562 
Su tüketimi yüksek olan bitki örtüsü altındaki derinliği fazla olmayan taban suyu seviyesinin günlük değişimi 10-15 cm kadar olabilmektedir. Eğimli dağ yamacı gibi arazilerde taban suyu seviyesinin mevsimlik değișimi daha fazla olup, birkaç santimetreden 10-15 m’ye kadar değişebilmektedir. Genel olarak, taban suyu seviyesinin çok yıllık değişimi, mevsimlik değişimden daha fazla olmaktadır. Taban suyu derinliğinin ve seviyesinin değişimi toprakların su, tuz bilançosuna ve sıcaklık rejiminin oluşumuna da önemli düzeyde etki yapmakta, bu değişimin belirlenmesi ise sulanan topraklarda drenaj-kollektor şebekesinin yapılması yöntemlerinin belirlenmesinde gerekli olmaktadır (Faibishenko, 1986; Kats ve Shestakov, 1992). Taban suyu yüzey biçiminin (eğrisinin) oluşumu, taban suyu tablasının değişimi, yağış ve sulama suyunun toprağa girişine $\left(\mathrm{k}_{\mathrm{i}}\right.$ ) (infiltrasyona), evapotranspirasyon ve drenaj akışı ile topraktan uzaklaşan suyun miktarına (q) bağlı olmaktadır. Deneysel olarak belirlenmiștir ki, taban suyu derinliğinin değiş̧imi $q / \mathrm{k}_{\mathrm{i}}$ oranı ile yaklaşık olarak orantılıdır. Vejetasyon döneminde taban suyu tablası daha fazla değişmekte, bitki-kök bölgesinde nem durumunu etkilemekte, toprak tekstürü, toprak sıcaklığının değişimi vb. bu değişime önemli düzeyde etki yapmaktadır (Childs, 1943; 1945a,b; Luthin, 1957; Iiyama ve ark., 2005; Nishida ve ark., 2009; Wu ve Zhuang, 2010; Luo ve ark., 2011; Pirastru ve Niedda, 2013; Melaku ve Wang, 2019). Taban suyunun derinde olmaması durumunda, yağıș, sulama ve yıkama sonucunda oluşan toprağın ıslanma derinliğinin (Li ve ark., 2016; Ekberli ve Gülser, 2018a) değişimi de taban suyu tablasının dalgalanmasına etki yapmaktadır.

Taban suyu tablasının değişiminin belirlenmesinde teorik ve deneysel yöntemler uygulanmaktadır. Teorik yöntemler, doğrusallaştırılmış Boussinesq denkleminin analitik veya yarı analitik, Richards denkleminin sayısal ve su bilançosu denkleminin çözümlerinden oluşmaktadır (Knotters ve Bierkens, 2000; Rai ve ark., 2006; Park ve Parker, 2008; Cuthbert, 2010; Neto ve ark., 2015; Jeong ve Park, 2017; Ekberli ve Gülser, 2018b; Hayek, 2019). Yeraltı hidroloji süreçlerinin sürekli olarak belirlenmesinin zor olduğu veya belirlenememesi durumlarında deneysel verilere bağlı olarak doğrusal veya doğrusal olmayan yöntemler uygulanmaktadır (Knotters ve Bierkens, 2000; Coulibaly ve ark., 2001; Coulibaly ve Baldwin, 2005; Okkonen ve Klöve, 2010; Yoon ve ark., 2011). Kong ve ark. (2015), taban suyu tablasının değişimine etki yapan faktörleri araştırarak, değişimin tahmin edilmesinde Boussinesq denklemini kullanmışlardır. Çok değişken yağışlı veya sulanan alanlardaki drenaj çalışmalarında, drenaj borularına giden geçici su akışı da Boussinesq denklemi ile tanımlanmaktadır (Singh ve ark., 2006). Boussinesq denkleminin analitik çözümlerinin temel dezavantajı, toprak homojenliği varsayımıdır (Singh ve ark., 1996). Bazı araştırmacılar başlangıç su tablasının parabolik veya eliptik şekilleri için tek boyutlu Boussinesq denkleminin çeşitli başlangıç ve sınır koşullarında analitik ve sayısal çözümlerini belirlemişlerdir (Dumm, 1954, 1964; Uziak ve Chieng, 1989; Singh et al., 2006). Mehdinejadiani ve Fathi (2020) tarafndan yapılan bir araştırmada, su tablasının parabolik ve eliptik şekilleri için su tablası yüksekliğinin belirlenmesinde kesirli uzaysal Boussinesq denkleminin çözümünden yararlanılmıştır. Su (2017) tarafından ise, yeraltı su akışının modellenmesinde, kesirli Boussinesq denklemi başlangıç ve sınır koşullarında uygulanmıştır. Zavala ve ark. (2007), laboratuvar koşullarında gerçekleştirilen bir drenaj denemesini tanımlamak için Boussinesq denkleminin sayısal çözümünü kullanmışlardır.

$\mathrm{Bu}$ çalışmanın amacı, Boussinesq denkleminin çözümüne bağlı olarak taban suyu tablası yüksekliğinin değişimine göre taban suyu seviyesinin incelenmesidir.

\section{Materyal ve Yöntem}

Kapalı olmayan geçirgen toprak katmanının birim kesit alanında gerçekleşen bir boyutlu su akışı, mesafeye $(\mathrm{x})$ ve zamana $(\mathrm{t})$ bağlı olan taban suyu tablasının $\mathrm{h}(\mathrm{x}, \mathrm{t})$ yüzey seviyesinin değişimini etkilemektedir. Bu durumda, Dupuit yaklaşımına (Dupuit,1863) ve Darcy yasasına (Darcy, 1856) bağlı olarak elde edilen Boussinesq denklemi (doğrusal olmayan difüzyon denklemi) aşağıdaki gibi ifade edilir (Boussinesq, 1904; Lockington ve ark., 2000; Tang ve Alshawabkeh, 2006; Telyakovskiy ve ark., 2016; Su, 2017; Ekberli ve Gülser, 2018b):

$$
\frac{\partial h}{\partial t}=\frac{k \rho g}{\mu \varphi} \frac{\partial}{\partial x}\left(h \frac{\partial h}{\partial x}\right)
$$

Burada, k -ortamın geçirgenlik katsayısı (gözenekli ortamda gerçekleşen akıma gösterilen direncin ölçütü); $\rho$-suyun yoğunluğu, $\mathrm{kgm}^{-3} ; g=9.81 \mathrm{~m} \mathrm{sn}^{-2}$-yerçekimi ivmesi; $\mu$-sıvının dinamik akışkanlık katsayısı; $\varphi$ çarpımı, ancak topraktaki gözeneklerin su ile doymuş durumda olduğunun göstergesidir. Kapalı olmayan 
geçirgen toprak katmanındaki bir boyutlu su akışı için, Dupuit yaklaşımı ve Darcy yasasına bağlı olarak elde edilen Boussinesq denklemi (doğrusal olmayan difüzyon denklemi) araștırmanın materyalini oluşturmaktadır. Çalışmanın gerçekleştirilmesinde matematiksel modelleme yöntemi kullanılmıştır.

\section{Bulgular ve Tartışma}

\section{Matematiksel Açıklama}

Toprağa giren ve uzaklaşan su miktarına bağlı olarak, $h(x, t)$ taban suyu seviyesi küçük değişime $\left(h_{d}\right)$ uğramakta ve bu durumda $h=h_{0}+h_{d}$ (2) olmaktadır (Şekil 1).

Burada, $h_{0}$-sabit taban suyu seviyesi ve $\left|h_{d}\right|<<h_{0}$.

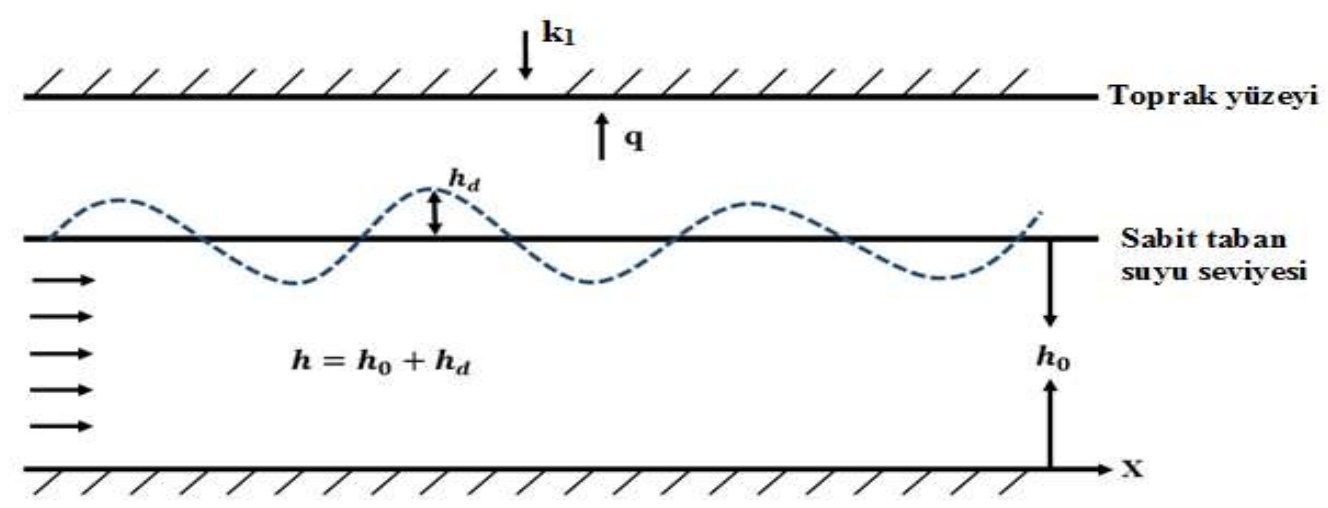

Şekil 1. Taban suyu tablasının ve seviyesinin değişimi

$k_{1}$ - infiltrasyon; $q$ - topraktan uzaklaşan su; $h_{0}$ - sabit taban suyu seviyesi; $h_{d}$ - taban suyu seviyesinin değişimi

(2) ifadesi (1)'de yerine konursa,

$$
\begin{aligned}
& \frac{\partial\left(h_{0}+h_{d}\right)}{\partial t}=\frac{k \rho g}{\mu \varphi} \frac{\partial}{\partial x}\left[\left(h_{0}+h_{d}\right) \frac{\partial}{\partial x}\left(h_{0}+h_{d}\right)\right]=\frac{k \rho g}{\mu \varphi} \frac{\partial}{\partial x}\left[\left(h_{0}+h_{d}\right) \frac{\partial h_{d}}{\partial x}\right]= \\
& \frac{k \rho g}{\mu \varphi} \frac{\partial}{\partial x}\left(h_{0} \frac{\partial h_{d}}{\partial x}+h_{d} \frac{\partial h_{d}}{\partial x}\right)=\frac{k \rho g}{\mu \varphi}\left(h_{0} \frac{\partial^{2} h_{d}}{\partial x^{2}}+\frac{\partial h_{d}}{\partial x} \frac{\partial h_{d}}{\partial x}+h_{d} \frac{\partial^{2} h_{d}}{\partial x^{2}}\right)
\end{aligned}
$$

bulunur.

$h_{d}$ 'ye göre kare terimin $\left(\frac{\partial h_{d}}{\partial x} \frac{\partial h_{d}}{\partial x}\right)$ ve $h_{d}$ 'nin küçük değerlere yaklaştı̆̆ı göz önüne alınarak, ilk terimle sinırlandırma yapılırsa (3) ifadesinden

$$
\frac{\partial h_{d}}{\partial t}=\frac{k \rho g h_{0}}{\mu \varphi} \frac{\partial^{2} h_{d}}{\partial x^{2}}
$$

elde edilir. (4) ifadesi, birçok araştırmacılar tarafından çeşitli yöntemlerle çözülen bir boyutlu ve sabit olmayan ısı iletkenliği denklemiyle benzerlik göstermektedir. Böyle ki,

$$
\frac{\partial T}{\partial t}=a^{2} \frac{\partial^{2} T}{\partial x^{2}} \quad(0 \leq x \leq \infty, t>0)
$$

ısı iletkenliğinin $T(0, t)=T_{o}+A \cos \omega t$ sınır koşulunda çözümü

$$
T(x, t)=T_{o}+A e^{-x \sqrt{\frac{\omega}{2 a^{2}}}} \cos \left(-x \sqrt{\frac{\omega}{2 a^{2}}}+\omega t\right) \text { veya }
$$




$$
T(x, t)=T_{o}+\frac{A}{e^{x \sqrt{\frac{\omega}{2 a^{2}}}}} \cos \left(-x \sqrt{\frac{\omega}{2 a^{2}}}+\omega t\right)
$$

biçiminde olmaktadır (Gülser ve Ekberli, 2002; Ekberli ve Gülser, 2014; Ekberli ve Sarılar, 2015; Gülser ve Ekberli, 2019).

(Burada, $T_{o}$-toprak yüzeyinin ortalama sıcaklığı, ${ }^{\circ} \mathrm{C} ; A$-toprak yüzeyindeki maksimum veya minimum sıcaklığın ortalama yüzey sıcaklığından olan farkı ifade eden amplitüt; $\omega=2 \pi / P$ ve $P$-periyot, yani dalganın bir döngüsünü tamamlaması için gerekli olan zamandır)

Taban suyunun kapalı olmayan yarı sonsuz kısmının başlangıcından $x>0$ mesafede, taban suyu tablasının değişimi $h_{d}$ 'nin periyodik dalgalanmasına bağlı olup, bu değişim $x=0$ 'da

$$
h_{d}=h_{d A} \cos \omega t
$$

(Burada, $h_{d A}$ taban suyu seviyesinin amplitütüdür) kuralı ile gerçekleşmektedir. Taban suyu tablasının (7) kuralı biçiminde harmonik değiş̧imi sulama veya yağmur sonucunda oluşmaktadır. Gözenekli ortamda (toprakta) akışı ifade eden (4) denklemindeki $\frac{k \rho g h_{0}}{\mu \varphi}$ parametresi, ısı iletkenliğinin (5) denklemindeki $a^{2}$ parametresi ile ekvivalentdir. Bu durumda, (5) denkleminim çözüm yöntemine bağlı ve (6) çözümüne benzer biçimde, (4) denkleminin (7) sınır koşulundaki çözümü aşağıdaki gibi olur:

$$
\begin{gathered}
h_{d}=h_{d A} e^{-x \sqrt{\frac{\omega \mu \varphi}{2 k \rho g h_{0}}}} \cos \left(\omega t-x \sqrt{\frac{\omega \mu \varphi}{2 k \rho g h_{0}}}\right) \text { veya } \\
h_{d}=\frac{h_{d A}}{x \sqrt[x]{\frac{\omega \mu \varphi}{2 k \rho g h_{0}}}} \cos \left(\omega t-x \sqrt{\frac{\omega \mu \varphi}{2 k \rho g h_{0}}}\right)
\end{gathered}
$$

$x=\sqrt{\frac{2 k \rho g h_{0}}{\omega \mu \varphi}}$ (9) olduğunda (8) ifadesinden $h_{d}=\frac{h_{d A}}{e}$ elde edilir. Bu ise, taban suyu tablasının dalgalanma amplitütünün (9) mesafesinde $e$ sayısı kadar azaldığını göstermektedir. Dolayısıyla, taban suyu tablasının dalgalanma amplitütü eksponansiyel olarak değişmektedir. Taban suyu tablasının dalgalanması durumunda (2) ifadesine göre, taban suyu seviyesi

$$
h=h_{0}+\frac{h_{d A}}{e^{x \sqrt{\frac{\omega \mu \varphi}{2 k \rho g h_{0}}}}} \cos \left(\omega t-x \sqrt{\frac{\omega \mu \varphi}{2 k \rho g h_{0}}}\right)
$$

olarak belirlenmektedir.

\section{Taban suyu tablasının ve seviyesinin mesafe ve zamana bağlı olarak değişimi}

Taban suyu tablasının değişimi taban suyu derinliğine, dolayısıyla toprak özelliklerinin değişimini etkilemekte; aynı zamanda sulanan topraklarda drenaj sisteminin yapılmasında gereklidir. Bu çalışmada, gözenekli ortamda akış denkleminin, ısı iletkenliği denkleminin analitik çözümüne benzer olarak elde edilen ifadeye göre taban suyu tablasının ve seviyesinin değişimi gösterilmiştir. Bu analitik ifade örnek bir çözüm ile açıklanmaya çalışılırsa, orta düzeyde geçirgenliğe sahip bir toprak ortamı için, bu toprak ortamında suyun akışkanlığının $\mu=1.14 \cdot 10^{-3} \mathrm{~kg} \mathrm{~m}^{-1} \mathrm{sn}^{-1}$; gözenekli ortamın geçirgenlik katsayısının (özgüsel iletkenliğinin) $k=10^{-12} \mathrm{~m}^{2}$; suyun yoğunluğunun $\rho=1000 \mathrm{~kg} \mathrm{~m}^{-3}$; yerçekimi ivmesinin $g=9.81 \mathrm{~m} \mathrm{sn}^{-2}$ ve suyla doygun gözenekliğin $\varphi=0.45$ varsayımı durumunda, $\quad \omega=\frac{2 \pi}{P}=\frac{6.28}{24 \text { saat }}=7.267 \cdot 10^{-5} \mathrm{sn}^{-1} \quad$ için $\frac{\omega \mu \varphi}{2 k \rho g h_{0}}=\frac{1.900 m^{-1}}{h_{0}}$ olarak (10) ifadesi aşă̆ıdaki gibi sadeleşir: 


$$
h=h_{0}+\frac{h_{d A}}{e^{x \sqrt{\frac{1.900 m^{-1}}{h_{0}}}}} \cos \left(7.267 \cdot 10^{-5} s n^{-1} \cdot t-x \sqrt{\frac{1.900 m^{-1}}{h_{0}}}\right)
$$

$h_{0}=2 m$ olarak, taban suyu tablası yüksekliğinin $\left(h_{d}\right)$ ve taban suyu seviyesinin $(h)$ mesafeye ve zamana bağlı olarak (11) ifadesine göre hesaplanan değerleri Çizelge 1'de verilmiştir.

Çizelge 1. Taban suyu tablası yüksekliği ve seviyesinin mesafeye ve zamana bağlı olarak değişimi ( $\left.\mathrm{h}_{\mathrm{d}} / \mathrm{h}\right)$.

\begin{tabular}{|c|c|c|c|c|c|c|c|c|}
\hline $\mathrm{x}, \mathrm{m}$ & \multicolumn{7}{|c|}{ Zaman (saat) } \\
\hline & 2 & 4 & 6 & 8 & 12 & 16 & 20 & 24 \\
\hline 0.5 & $\frac{0.123}{2.123}$ & $\frac{0.104}{2.104}$ & $\frac{0.058}{2.058}$ & $\frac{-0.004}{1.996}$ & $\frac{-0.108}{1.892}$ & $\frac{-0.104}{1.896}$ & $\frac{0.004}{2.004}$ & $\frac{0.108}{2.108}$ \\
\hline 1.0 & $\frac{0.068}{2.068}$ & $\frac{0.075}{2.075}$ & $\frac{0.063}{2.063}$ & $\frac{0.033}{2.033}$ & $\frac{-0.042}{1.958}$ & $\frac{-0.075}{1.925}$ & $\frac{-0,033}{1,967}$ & $\frac{0.042}{2.042}$ \\
\hline 1.5 & $\frac{0.027}{2.027}$ & $\frac{0.042}{2.042}$ & $\frac{0.046}{2.046}$ & $\frac{0.037}{2.037}$ & $\frac{-0.005}{1.995}$ & $\frac{-0.042}{1.958}$ & $\frac{-0.038}{1.962}$ & $\frac{0.005}{2.005}$ \\
\hline 2.0 & $\frac{0.004}{2.004}$ & $\frac{0.018}{2.018}$ & $\frac{0.026}{2.026}$ & $\frac{0.028}{2.028}$ & $\frac{0.011}{2.011}$ & $\frac{-0.018}{1.982}$ & $\frac{-0.028}{1.972}$ & $\frac{-0.011}{1.989}$ \\
\hline 2.5 & $\frac{-0.006}{1.994}$ & $\frac{0.003}{2.003}$ & $\frac{0.011}{2.011}$ & $\frac{0.017}{2.017}$ & $\frac{0.013}{2.03}$ & $\frac{-0.003}{1.997}$ & $\frac{-0.016}{1.984}$ & $\frac{-0.013}{1.987}$ \\
\hline
\end{tabular}

Çizelge 1'den görüldüğü gibi, taban suyu tablası yüksekliği ve taban suyu seviyesi mesafeye ve zaman bağlı olarak değişmekte, mesafe arttıkça bu değişim azalmaktadır. Başlangıç saatte azalma, sonra artma ve tekrar azalma eğilimi gerçekleşmektedir. $2.0 \mathrm{~m}$ ve $2.5 \mathrm{~m}$ mesafede değişim daha da azalmakta, dolayısıyla taban suyu tablasının dalgalanması "sönme" sürecine yaklaşmaktadır. Tüm mesafelerde taban suyu tablasının değişimi harmonik fonksiyon biçiminde olmaktadır. Bitki örtüsü, sulama ve yağmur miktarı, toprak bünyesi, arazinin drenaj durumu, infiltrasyon (ki) ile evapotranspirasyon ve drenaj akışı ile topraktan uzaklaşan su miktarının (q) oranı (q/ki) yeraltı su akışı vb. gibi faktörler taban suyu tablası yüksekliğine ve taban suyu seviyesine etki yapmaktadır. Luthin (1957) tarafından deneysel olarak gösterilmiștir ki, taban suyu tablasının yüksekliğinin, dolayısıyla taban suyu seviyesinin değiş̧imi $\mathrm{q} / \mathrm{k}_{\mathrm{i}}$ değeriyle yaklaşık olarak orantılıdır ve taban suyunun yüzey eğri biçiminin oluşumunda temel parametredir. Kats ve Shestakov (1992)'a göre ise, su tüketimi yüksek olan bitki örtüsü altındaki derinliği fazla olmayan taban suyu seviyesinin günlük değişimi 10-15 cm kadar olabilmektedir. Eğimli dağ yamacı gibi arazilerde taban suyu seviyesinin mevsimlik değişimi daha fazla olup, birkaç santimetreden 10-15 m'ye kadar değişebilmektedir. $\mathrm{Bu}$ çalışmada gözenekli ortamda akış denkleminin kullanılan çözümü, harmonik (kosnüsoid) fonksiyonla ifade edilen sınır koşuluna göre belirlendiği için, taban suyu tablasının ve taban suyu seviyesinin değişimini kısa süreçlerde (<3-4 gün) ifade edebilmektedir. Farklı sınır koşullarında çözümün, dolayısıyla araştırmanın daha da genelleştirilmesi mümkün gözükmektedir.

\section{Sonuç}

Dupuit yaklaşımına ve Darcy hızına bağlı olarak elde edilen Boussinesq denklemine (doğrusal olmayan difüzyon denklemi) bağlı olarak, taban suyu tablası yüksekliğinin değişiminin matematiksel modellenmesi ve taban suyu seviyesinin değerlendirilmesi incelenmiştir. Taban suyu tablası yüksekliğinin değişimini ifade eden denklem, çeşitli yöntemlerle çözülen bir boyutlu ve sabit olmayan ısı iletkenliği denklemiyle benzer olmaktadır. Teorik ve sayısal sonuçlar, taban suyu tablası dalgalanmasının periyodik ve asimetrik olduğunu göstermektedir. Mesafe artışına bağlı olarak, taban suyu tablası dalgalanmalarının genliği azalmakta; faz gecikmeleri gerçekleşmektedir. Taban suyu tablasının dalgalanma amplitütü eksponansiyel olarak değişmektedir. Taban suyu tablası yüksekliğinin değişiminin belirlenmesi, taban suyu seviyesinin tahmin edilmesinde temel faktörlerden biridir. Yeraltı ve yüzey su kaynaklarının yönetiminde, toprakların sulama ve yıkama su miktarlarının belirlenmesinde vb. taban suyu seviyesinin değişimiyle birlikte taban suyu tablasının dalgalanmasının da göz önüne alınması gereklidir. Taban suyu tablası yüksekliğinin değişimi, topraktaki toplam buharlaşmaya, toprakların fiziksel ve kimyasal özelliklerine, su-tuz dengesine, isı transferine, bitki kök bölgesindeki nem değişimine vb. önemli düzeyde etki yapmaktadır. 


\section{Kaynaklar}

Boussinesq MJ, 1904. Recherches theoriques sur l'ecoulement des nappes d'eau infiltrées dans le sol et sur debit de sources. Journal de Mathématiques Pures et Appliquées 10: 5-78.

Childs EC, 1943. The water table, equipotentials, and streamlines in drained land. Soil Science, 56(5): 317-330.

Childs EC, 1945a. The water table , equipotentials, and streamlines in drained land: II. Soil Science, 59(4): 313-328.

Childs EC, 1945b. The water table, equipotentials, and streamlines in drained land: III. Soil Science, 59(5): 405-415.

Coulibaly P, Anctil F, Aravena R, Bobee B, 2001. Artificial neural network modeling of water table depth fluctuations. Water Resources Research 37 (4): 885-896.

Coulibaly P, Baldwin CK, 2005. Nonstationary hydrological time series forecasting using nonlinear dynamic methods. Journal of Hydrology 307 (1): 164-174.

Cuthbert MO, 2010. An improved time series approach for estimating groundwater recharge from groundwater level fluctuations. Water Resources Research 46 (9): W09515.

Darcy H, 1856. Les fontaines publiques de la ville de Dijon. Dalmont, Paris, 647 p.

Dumm LD, 1954. Drain spacing formula: new formula for determining depth and spacing of subsurface drains in irrigated lands. American Society of Agricultural Engineers, 35: 726-730.

Dumm LD, 1964. Transient flow concept in subsurface drainage: its validity and use. Transactions of the American Society of Agricultural Engineers, 7: 142-146.

Dupuit J, 1863. Études théoriques et pratiques sur le mouvement des eaux dans les canaux découverts et a travers les terrains perméables. Dunod, Paris, 364 p.

Ekberli İ, Gülser C, 2014. Estimatıon of soil temperature by heat conductivity equation. Vestnik Bashkir State Agrarian University (Вестник Башкирского Государственного Аграрного Университета), 2 (30):12-15.

Ekberli İ, Gülser C, 2018a. Sulamada toprağın ıslanma derinliğinin belirlenmesi. Anadolu Tarım Bilimleri Dergisi, 33(2): 142-148.

Ekberli İ, Gülser C, 2018b. Boussinesq denkleminin çözümüne bağlı olarak taban suyu seviyesi yüksekliğinin incelenmesi. Toprak Bilimi ve Bitki Besleme Dergisi, 6(2): 134-142.

Ekberli İ, Sarılar Y, 2015. Toprak sıcaklığının profil boyunca sönme derinliğinin ve gecikme zamanının belirlenmesi. Ege Üniversitesi Ziraat Fakültesinin Dergisi, 52 (2): 219-225.

Faibishenko BA, 1986. Water-salt rejime of soils under irrigation. Agropromizdat, Moscow (in Russian), 304 p.

Gülser C, Ekberli İ, 2002. Toprak sıcaklığının profil boyunca değişimi. Ondokuz Mayıs Üniversitesi Ziraat Fakültesinin Dergisi, 17(3): 43-47.

Gülser C, Ekberli İ, 2019. Toprak sıcaklığının tahmininde ısı taşınım denklemi ve pedotransfer fonksiyonun karşılaştırılması. Toprak Bilimi ve Bitki Besleme Dergisi, 7(2): 158-166.

Hayek M, 2019. Accurate approximate semi-analytical solutions to the Boussinesq groundwater flow equation for recharging and discharging of horizontal unconfined aquifers. Journal of Hydrology, 570: 411-422.

Iiyama I, Fujimoto T, Nagata O, Hasegawa S, 2005. Formation of a groundwater table by trench irrigation and evapotranspiration in a drained peatland. Soil Science \& Plant Nutrition, 51(3): 313-322.

Jeong J, Park E, 2017. A shallow water table fluctuation model in response to precipitation with consideration of unsaturated gravitational flow. Water Resources Research 53: 3505-3512.

Kats DM, Shestakov VM, 1992. Melioration hydrogeology. Moscow State University Press, Mockow (in Russian), pp.7192.

Knotters M, Bierkens MFP, 2000. Physical basis of time series models for water table depths. Water Resources Research 36 (1): 181-188.

Kong J, Xin P, Hua G-F, Luo ZY, Shen C-J, Chen D, Li L, 2015. Effects of vadose zone on groundwater table fluctuations in unconfined aquifers. Journal of Hydrology 528: 397-407.

Li X, Jin M, Zhou N, Huang J, Jiang S, Telesphore H, 2016. Evaluation of evapotranspiration and deepper colation under mulched drip irrigation in an oasis of Tarimbasin, China. Journal of Hydrology, 538: 677-688.

Lockington DA, Parlange J.-Y, Parlange MB, Selker J, 2000. Similarity solution of the Boussinesq equation. Advances in Water Resources 23: 725-729.

Luo JM, Yang F, Wang YJ, Ya YJ, Deng W, Zhang XP, Liu Z, 2011. Mechanism of soil sodification at the local scale in Songnen Plain, Northeast China, as affected by shallow groundwater table. Arid Land Research and Management, 25(3): 234-256.

Luthin JN (Editor), 1957. Drainage of Agricultural Lands. Agronomy Monographs, 7, American Society of Agronomy, Crop Science Society of America, and Soil Science Society of America, Publisher Madison, Wisconsin, 620 p.

Mehdinejadiani B, Fathi P, 2020. Analytical solutions of space fractional Boussinesq equation to simulate water table profiles between two parallel drainpipes under different initial conditions. Agricultural Water Management, 240: 106324.

Melaku ND, Wang J, 2019. A modified SWAT module for estimating groundwater table at Lethbridge and Barons, Alberta, Canada. Journal of Hydrology, 575: 420-431.

Neto DC, Chang HK, van Genuchten MT, 2015. A mathematical view of water table fluctuations in a shallow aquifer in Brazil. Groundwater, 54 (1): 82-91. 
Nishida K, Khan NM, Shiozawa S, 2009. Effects of salt accumulation on the leaf water potential and transpiration rate of pot-grown wheat with a controlled saline groundwater table, Soil Science and Plant Nutrition, 55(3): $375-384$.

Okkonen J, Klöve B, 2010. A conceptual and statistical approach for the analysis of climate impact on ground water table fluctuation patterns in cold conditions. Journal of Hydrology, 388. 1-12.

Park E, Parker JC, 2008. A simple model for water table fluctuations in response to precipitation. Journal of Hydrology 356 (3): 344-349.

Pirastru M, Niedda M, 2013. Evaluation of the soil water balance in an alluvial flood plain with a shall ow groundwater table. Hydrological Sciences Journal, 58 (4): 898-911.

Rai SN, Manglik A, Singh VS, 2006. Water table fluctuation owing to time-varying recharge pumping and leakage. Journal of Hydrology 324 (1-4): 350-358.

Singh MP, Chauhan HS, Ram S, 1996. Unsteady state drainage in a vertically heterogeneous soil. Agricultural Water Management, 31: 285-293.

Singh RM, Singh KK, Singh SR, 2006. Falling water tables in a sloping/nonsloping aquifer under various initial water table profiles. Agricultural Water Management, 82(1-2): 210-222.

$\mathrm{Su}, \mathrm{N}$., 2017. The fractional Boussinesq equation of groundwater flow and its applications. Journal of Hydrology, 547: 403-412.

Tang G, Alshawabkeh AN, 2006. A semi-analytical time integration for numerical solution of Boussinesq equation. Advances in Water Resources,29: 1953-1968.

Telyakovskiy AS, Kurita S, Allen MB, 2016. Polynomial-based approximate solutions to the Boussinesq equation near a well. Advances in Water Resources 96: 68-73.

Uziak J, Chieng S, 1989. Drain-spacing formula for transient state flow with ellipse as an initial condition. Canadıan Agricultural Engineering, 31: 101-105.

Wu L, Zhuang S, 2010. Experimental investigation of effect of tide on coastal groundwater table. Journal of Hydrodynamics, 22(1): 66-72.

Yoon H, Jun SC, Hyun Y, Bae GO, Lee KK, 2011. A comparative study of artificial neural networks and support vector machines for predicting groundwater levels in a coastal aquifer. Journal of Hydrology 396 (1): 128-138.

Zavala M, Fuentes C, Saucedo H, 2007. Non-linear radiation in the Boussinesq equation of the agricultural drainage. Journal of Hydrology, 332: 374-380. 